\title{
The Effect of GCSB-5 a New Herbal Medicine on Changes in Pain Behavior and Neuroglial Activation in a Rat Model of Lumbar Disc Herniation
}

\author{
Hee Kyung Cho, M.D., Ph.D., ${ }^{1}$ So-Yeon Kim, Ph.D., ${ }^{2}$ Mi Jung Choi, ${ }^{2}$ Seung Ok Baek, M.D., ${ }^{3}$ Sang Gyu Kwak, Ph.D., ${ }^{4}$ \\ Sang Ho Ahn, M.D., Ph.D., \\ Departments of Physical Medicine and Rehabilitation, ${ }^{1}$ Medical Statistics, ${ }^{4}$ College of Medicine, Catholic University of Daegu, Daegu, Korea \\ Medical Devices Clinical Trial Center, ${ }^{2}$ Department of Physical Medicine and Rehabilitation, ${ }^{3}$ College of Medicine, Yeungnam University, \\ Daegu, Korea
}

\begin{abstract}
Objective : Lumbar disc herniation can induce sciatica by mechanical compression and/or chemical irritation. The aim of this study was to compare the effects of GCSB-5 (Shinbaro ${ }^{\circledR}$ ) and NSAIDs on pain-related behavior and on the expressions of microglia, astrocytes, CGRP, TRPV1, IL-6, and CX3CL1 in a rat model of lumbar disc herniation.

Methods : 112 male Sprague-Dawley rats underwent implantation of nucleus pulposus to a dorsal root ganglion (DRG). Rats were divided into five groups as follows; a saline group (the vehicle control group) ( $n=27)$, a $10 \mathrm{mg} / \mathrm{kg}$ aceclofenac group (the aceclofenac group) ( $\mathrm{n}=22$ ), and 100, 300 or 600 mg/kg GCSB-5 groups (the GCSB-5 100, 300, or 600 groups) (n=21 for each group). Rats were tested for mechanical allodynia at 3 days after surgery and at 1 day, 3 days, 7 days, 14 days, 21 days, 28 days, 35 days, 42 days, 49 days, and 56 days after treatment commencement. Immunohistochemical staining of microglia (Iba1), astrocytes (GFAP), CGRP, and TRPV1, and PCR for IL-6 and CX3CL1 were performed on spinal dorsal horns and DRGs at 56 days after medication commencement.

Results : After 56 days of GCSB-5 300 administration, mechanical withdrawal thresholds were significantly increased $(p<0.05)$, and immunohistochemical expressions of Iba1, GFAP, CGRP, and TRPV1 were reduced than other groups, but this difference was not statistically significant. Conclusion : These results indicate GCSB-5 reduces mechanical allodynia and downregulates neuroglial activity and the expressions of CGRP and TRPV1 in the spinal segments of a rat model of lumbar disc herniation.
\end{abstract}

Key Words : GCSB-5 · Lumbar disc herniation · Neuropathic pain · Microglia · Astrocytes · Calcitonin gene-related peptide.

\section{INTRODUCTION}

Lumbar disc herniation can injure spinal nerve roots and cause severe radicular pain, characterized by hyperalgesia, allodynia, decreased conduction velocity, and histologic changes ${ }^{34)}$. Previous studies have suggested that the clinical signs of lumbar disc herniation are caused by chemical factors released from the nucleus pulposus and by mechanical compression of lumbar nerve roots $^{2,21,32-34,48)}$. Proinflammatory cytokines, such as, tumor necrosis factor-alpha (TNF- $\alpha$ ), interleukin-1-beta (IL-1 $\beta$ ), and interleukin-6 (IL-6), and nitric oxide and phospholipase A2 are known as chemical inducers of sciatica ${ }^{3,4,15,20,33)}$. Nucleus pulposus contains TNF- $\alpha$, and when nucleus pulposus is applied to a dorsal root ganglion (DRG) it induces pain-related behavior and causes morphological and functional changes in DRGs and spinal cords in rats ${ }^{15,32-34,38)}$. Various medications are used to treat radicular pain. Non-steroidal anti-inflammatory drugs (NSAIDs) are generally used as analgesics, and most of the analgesic effects of anti-inflammatory drugs are due to blocking of the syntheses of inflammatory products from arachidonic acid. However, this interruption can generate undesirable adverse drug reactions, such as, gastrointestinal and cardiovascular problems ${ }^{43}$.

GCSB-5 (Shinbaro ${ }^{\circledR}$; Green Cross Corp., Yongin, Korea) is a medicine prepared from six herbs (Ledebouriellae Radix, Achyranthis Radix, Acanthopanacis Cortex, Cibotii Rhizoma, Glycine Semen, and Eucommiae Cortex), which are used in traditional East Asian medicine to treat osteoarthritis. A mixture containing fixed ratios of the six herbs was powdered and boiled

- Received : September 11, 2015 • Revised : September 24, 2015 •Accepted : February 10, 2016

- Address for reprints : Sang Ho Ahn, M.D., Ph.D.

Department of Physical Medicine and Rehabilitation, College of Medicine, Yeungnam University, 170 Hyeonchung-ro, Nam-gu, Daegu 42415, Korea

Tel : +82-53-620-3268, Fax : +82-53-624-8356, E-mail : spineahn@ynu.ac.kr

- This is an Open Access article distributed under the terms of the Creative Commons Attribution Non-Commercial License (http://creativecommons.org/licenses/by-nc/3.0) which permits unrestricted non-commercial use, distribution, and reproduction in any medium, provided the original work is properly cited. 
in distilled water. The resulting extract was ultrafiltered to exclude the components with molecular weights exceeding 10000. The extract was then dried to a powder by freeze drying. A capsule of GCSB- 5 contains $300 \mathrm{mg}$ of the dried extract ${ }^{25}$. Although the active ingredients of GCSB- 5 have not been completely identified, recent studies have described the analgesic and antiinflammatory effects of Ledebouriellae Radix ${ }^{24)}$, the antioxidant and anti-inflammatory effects of Achyranthis Radix ${ }^{14)}$, and the antioxidant and analgesic effects of Eucommiae Cortex ${ }^{12)}$. However, mechanistic evidence regarding molecular changes in spinal segments induced by GCSB- 5 after lumbar disc herniation is lacking, and the comparative effects of GCSB-5 and NSAIDs on radicular pain have not been well studied.

This study was undertaken to compare the effects of aceclofenac (a NSAID) and GCSB-5 on pain-related behavior and on the expressions of Ionized calcium-binding adapter molecule 1 (Iba1), Glial fibrillary acidic protein (GFAP), calcitonin gene-related peptide (CGRP), transient receptor potential vanilloid 1 (TRPV1), IL-6, and CX3CL1 in a rat model of lumbar disc herniation.

\section{MATERIALS AND METHODS}

\section{Animals}

A total of 112 female Sprague-Dawley rats (200-220 g) were used. The animals were housed in plastic cages at room temperature under a 12-h light-dark cycle with free access to food and water. All experiments were conducted in a humane manner in accordance with the guidelines issued by the Institutional Animal Care and Use Committee of Yeungnam University, Korea.

\section{Lumbar disc herniation procedures}

Rats were anesthetized with an intraperitoneal injection of zoletil (Virbac; $50 \mathrm{mg} / \mathrm{kg}$ ). With an animal placed prone, an incision of $\sim 1 \mathrm{~cm}$ was made on the dorsal surface of the proximal tail for autologous nucleus pulposus harvesting. The disc between the second and third coccygeal vertebrae was incised, and nucleus pulposus was harvested by curette. A midline dorsal incision was then made over the lumbar spine, multifidus muscles were separated along the L4-S1 spinous processes, and left L5 nerve roots and DRGs were exposed by laminectomy. The harvested nucleus pulposus was then implanted next to the left L5 nerve root just proximal to DRG without applying mechanical compression. Similar amounts of nucleus pulposus were implanted in an identical manner in all animals.

\section{Treatment after nucleus pulposus implantation}

After nucleus pulposus implantation animals were divided into five treatment groups : a saline group (the vehicle control group) ( $\mathrm{n}=27)$, a $10 \mathrm{mg} / \mathrm{kg}$ aceclofenac group (the aceclofenac group) ( $\mathrm{n}=22$ ), and 100, 300, and $600 \mathrm{mg} / \mathrm{kg}$ GCSB-5 groups (the GCSB-5 100, 300, and 600 groups) ( $n=21$ for each group). Drugs were dissolved in $0.9 \%$ saline for administration. The drug concentrations used were based on clinical levels. Animals received $1.5 \mathrm{~mL}$ of the designated treatment solution orally once daily for 8 weeks beginning 3 days after surgery.

\section{Pain behavior evaluation}

Mechanical sensitivities of the plantar surfaces of ipsilateral hind paws were tested at 3 days after surgery and at 1 day, 3 days, 7 days, 14 days, 21 days, 28 days, 35 days, 42 days, 49 days, and 56 days after treatment commencement (Fig. 1). Mechanical allodynia was determined by measuring withdrawal response to mechanical stimulation of ipsilateral hind paws with von Frey filaments (North Coast Medical Inc., Gilroy, CA, USA), which had been calibrated in grams. Rats were placed in a clear plastic cage with a metal mesh floor, adapted to the testing environment for 30 minutes, and then the plantar surface of each hind paw was stimulated to cause slight filament bending for $5 \mathrm{sec}$ onds. Testing was started using a $1.4 \mathrm{~g}$ probe, and probe resistance was increased until a filament produced a consistent withdrawal response to more than 3 of 5 stimuli. Filaments were applied in increasing and decreasing thicknesses. $50 \%$ probability thresholds of mechanical paw withdrawal were then calculated. If no withdrawal response was elicited by the 26 -g filament, a mechanical threshold of $26 \mathrm{~g}$ was assigned.

\section{Immunohistochemical examination}

To study microglial and astrocyte activations in dorsal horns and CGRP and TRPV1 expressions in DRGs, we euthanized 4-6

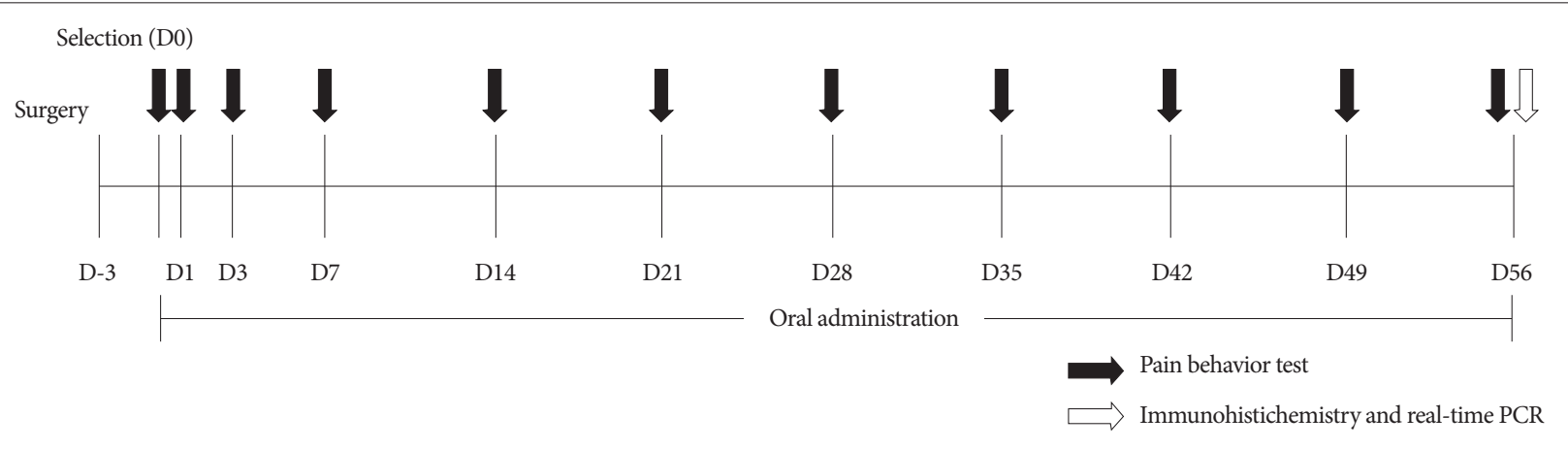

Fig. 1. Study flow schematic. 
rats per group at 56 days after treatment commencement (Fig. 1). Under anesthesia, a catheter was inserted into the left ventricle, which was then rinsed with $500 \mathrm{~mL}$ of saline followed by $500 \mathrm{~mL}$ of $4 \%$ paraformaldehyde [in $0.1 \mathrm{~N}$ phosphate buffer (PB)]. The L5 spinal cord segment was removed, post-fixed for 2 days in the same fixative, and then were stored in 30\% sucrose (in PB) for at least 24 hours. Transverse sections of spinal cord and of DRGs (30 and $20 \mu \mathrm{m}$ thick, respectively) were prepared using a cryostat (Leica, Wetzlar, Germany) and stored in PB. All incubation and reaction procedures for multiple immunohistochemical staining were conducted at room temperature on a shaker. To enhance tissue penetration by antibodies, DRG sections were reacted with $50 \%$ ethanol for 30 minutes and rinsed with phosphate buffered saline (PBS) for $3 \times 5$ minutes and blocked to prevent nonspecific primary antibody reactions. Samples were then treated with $10 \%$ normal donkey serum (NDS; Jackson Immunoresearch, Westgrove, PA, USA). Tissue sections were incubated overnight in a mixture of primary antibodies, that is, mouse anti-ionized calcium-binding adapter molecule 1 (Iba1) (Wako, Osaka, Japan; 1 : 1000), mouse antiglial fibrillary acidic protein (GFAP) (BD Pharmingen, San Jose, CA, USA; $1: 100$ ), anti-transient receptor potential vanilloid type 1 (TRPV1) (Neuromics, Edina, MN, USA; 1 : 5000), and anti-calcitonin gene related peptide (CGRP) (Enzo, Farmingdale, NY, USA; $1: 200)$. After reaction completion, tissues were rinsed with PBS ( $3 \times 5$ minutes), treated with $2 \%$ NDS for 15 minutes, and incubated with cy3-conjugated donkey anti-mouse (Jackson Immunoresearch, PA, USA; $1: 100$ ), cy3-conjugated donkey antigoat (Jackson Immunoresearch, PA, USA; 1 : 100), and Alexa 488-conjugated donkey anti-rabbit (Invitrogen, Eugene, OR, USA; $1: 200$ ) antibodies for 3 hours, rinsed with PBS, and mounted with Vectashield (Vector Lab, Burlingame, CA, USA). All antibodies were tested for sensitivity and specificity beforehand. The dilutions used were optimal, according to the manufacturers' recommendations. Immunofluorescent images were acquired using a cooled charge-coupled device (CCD) camera (Olympus DP71, Tokyo, Japan) attached to a light microscope (Olympus BX51, Tokyo, Japan).

\section{Quantitative image analysis}

To quantify immunostaining of Ibal and GFAP in dorsal horns and CGRP and TRPV1 in DRGs, images were obtained of five spinal cord sections (for both Ibal and GFAP) and of five DRG sections from L5 per rat. One image $(898 \times 660 \mathrm{~mm})$ was taken with a CCD camera using the same shutter speed and digital gain of each spinal cord section. Images were encoded to blind the investigator before analysis. Pixels positive immunoreactive Ibal or GFAP were identified by applying an appropriate threshold gray value, and area fractions (immunopositive area/total frame area) were calculated using image analysis software (Leica application suite V4.2, Leica Microsystems, Heerbrugg, Switzerland). Numbers of CGRP- and TRPV1-postive DRG cells were counted using the image analysis software at the same time.

\section{Real-time polymerase chain reaction}

Real-time polymerase chain reaction (PCR) was performed on spinal cords and DRGs to assess the messenger RNA (mRNA) expressions of IL-6 and CX3CL1. 3-4 rats per group were euthanized at 56 days after treatment commencement and L5 spinal cord segments were divided into left (ipsilateral) and right (contralateral) halves, which included ventral and dorsal gray and white matter (Fig. 1). Total RNA was isolated from both halves of spinal cords and DRGs using Trizol reagent (Invitrogen Corp., Carlsbad, CA, USA) and was conducted using $1 \mathrm{mg}$ aliquots of total mRNA at $45^{\circ} \mathrm{C}$ using the High capacity cDNA reverse transcription kit (Applied Biosystems Inc., Foster City, CA, USA). Real-time PCR was performed using a Real Time ABI 7500 system (Applied Biosystems Inc., Foster City, CA, USA). Primers and a TaqMan probe were designed using ProbeFinder software (Universal Probe Library, UPL, Roche, Switzerland).

The following primers were used : 5'-TCCACTATCAACT GAACCAGGA-3' (sense) and 5'TTGGGTCAGCACAGAAGT GT-3' (antisense) for CX3CL1 (GenBank accession number : NM134455), 5'-TATGAACAGCGATGACCACTG-3' (sense) and 5'-TTGCTCTGAATGACTCTGGCTT-3' (antisense) for IL-6, and 5'-ACCACCATGGAGAAGGCTGG-3' (sense) and 5'-CTCAGTGTAGCCCAGGATGC-3' (antisense) for hypoxanthine phosphoribosyl-transferase (HPRT, GenBank accession number : NM012583.2). HPRT gene was used as an internal reference control. The master mix used consisted of $10 \mu \mathrm{mol} / \mathrm{L}$ of UPL probe, $10 \mu \mathrm{mol} / \mathrm{L}$ of each primer (sense and antisense), $10 \mu \mathrm{L}$ of $2 \times$ TaqMan master, and $2 \mu \mathrm{L}$ of cDNA. All PCR reactions were run in duplicate. After preincubation at $95^{\circ} \mathrm{C}$ for 10 minutes, PCR was performed over 50 cycles of denaturation at $95^{\circ} \mathrm{C}$ for $10 \mathrm{~s}$ and annealing at $60^{\circ} \mathrm{C}$ for $30 \mathrm{~s}$.

\section{Statistical analysis}

Outcomes were using descriptive analysis. Quantitative variables are presented as means (SDs). Paw withdrawal thresholds by time after treatment commencement, group, and interaction effects (time difference by group) were analyzed using repeated measures two factor analysis and multiple comparisons, and the results obtained were compared. One-way ANOVA was used to determine the significances of variable differences between D0 (the day of treatment commencement) and later times, and Scheffe test was used for multiple comparisons. $p$-values are presented for significant variables. All tests were 2 -sided and $p$-values of $<0.05$ were considered significance. The analysis was conducted using IBM SPSS ver. 19.0.

\section{RESULTS}

\section{Pain behavior}

For rats in the GCSB-5 300 group, mechanical allodynia of ipsilateral hind paws was significantly attenuated at 56 days after treatment commencement (treatment day 56, TD56) than in vehicle control group $(p<0.05)$. However, mechanical withdraw- 
al thresholds were not significantly in the aceclofenac and vehicle control groups on TD56 (Fig. 2).

\section{Microglia, Astrocytes, CGRP, and TRPV1}

Immunohistochemical examination of in GCSB-5 groups for Ibal and GFAP in dorsal horns, CGRP and TRPV1 in DRGs on TD56 revealed that numbers of microglia and astrocytes were

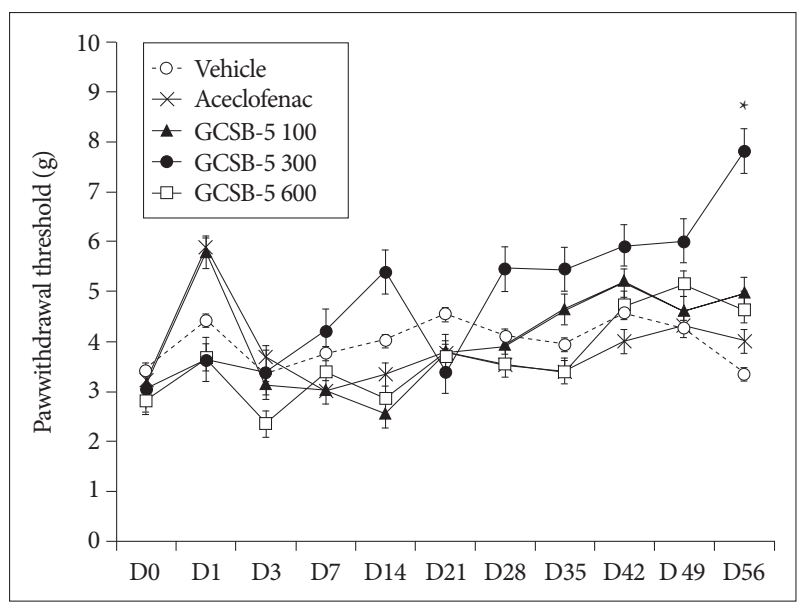

Fig. 2. Changes in mechanical withdrawal thresholds after drug or saline treatment in a rat model of lumbar disc herniation. GCSB-5 or aceclofenac was administered from 3 days after surgery. A significant increase in mean ipsilateral paw withdrawal threshold was found at 56 days after the commencement of GCSB- 5300 treatment versus vehicle controls. * Significantly different from vehicle group, $p<0.05$.
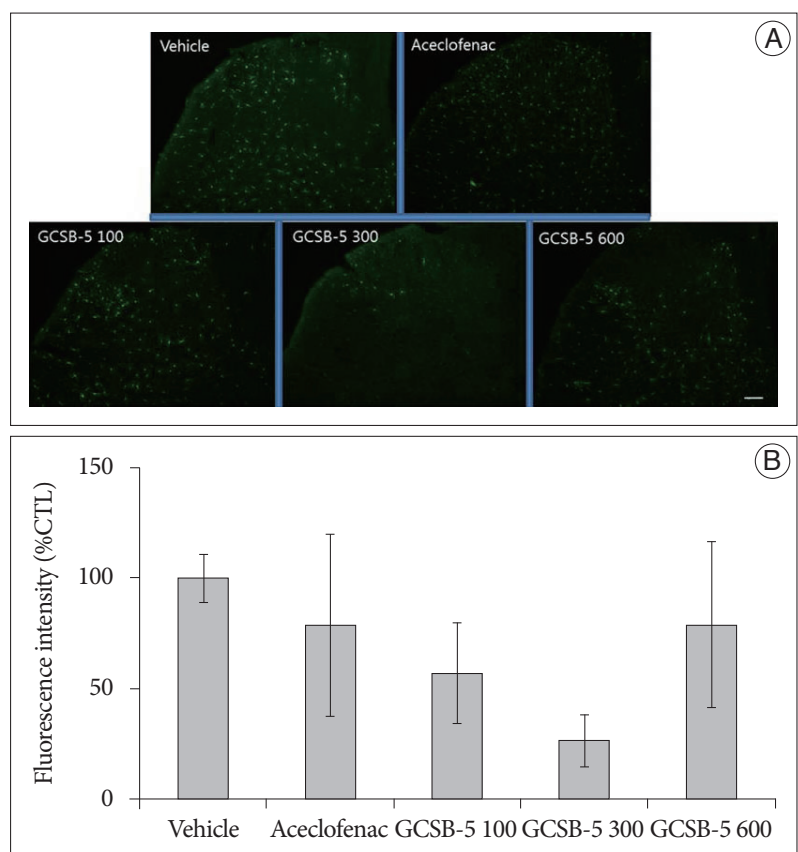

Fig. 3. Immunohistochemical expression of lba1 in the ipsilateral L5 dorsal horn $(A)$ and intensity of lba1 immunoreaction versus vehicle controls (B) at 56 days after treatment commencement. A : Iba1-positive microglia numbers were lower in the ipsilateral dorsal horns of the GCSB-5 300 group than in the aceclofenac group or vehicle controls. $B$ : the percentage decrease was greater in the GCSB- 5300 group than in the GCSB-5 100 , GCSB-5 600, aceclofenac or vehicle control groups. Results are presented as mean \pm SEM. Bar $=100 \mu \mathrm{m}$. attenuated in ipsilateral L5 dorsal horns and that CGRP and TRPV1 expressions were also attenuated in ipsilateral L5 DRGs versus vehicle controls. For all the proteins examined, the largest decreases were observed in the GCSB- 5300 group, but these were not statistically significant. In L5 dorsal horns, decreases in Ibal immunoreactivity observed on TD56 were; $21 \%$ in the aceclofenac group and 43\%, 73\%, and $21 \%$ in the GCSB-5 100 , 300, 600 groups versus the vehicle controls (Fig. 3). Corresponding decreases in GFAP immunoreactivity were 73\%, 67\%, 82\%, and $63 \%$, respectively (Fig. 4), decreases in CGRP were $21 \%$, $54 \%, 77 \%$, and $51 \%$ (Fig. 5), and decreases in TRPV1 were $7 \%$, $38 \%, 55 \%$, and $34 \%$, respectively (Fig. 6).

\section{Expressions of IL-6 and CX3CL1 mRNA}

The expressions of IL- 6 mRNA in the aceclofenac and three GCSB-5 groups were not significantly different from those of vehicle controls on TD56, and at this time CX3CL1 immunoreactivity was also unaffected by aceclofenac or GCSB-5 (Fig. 7).

\section{DISCUSSION}

In the present study, we compared the effects of GCSB-5 and of aceclofenac (a NSAID) on pain-related behavior and neuroglial expression in a rat model of lumbar disc herniation. On TD56, mechanical withdraw thresholds in the GCSB-5 300 group were
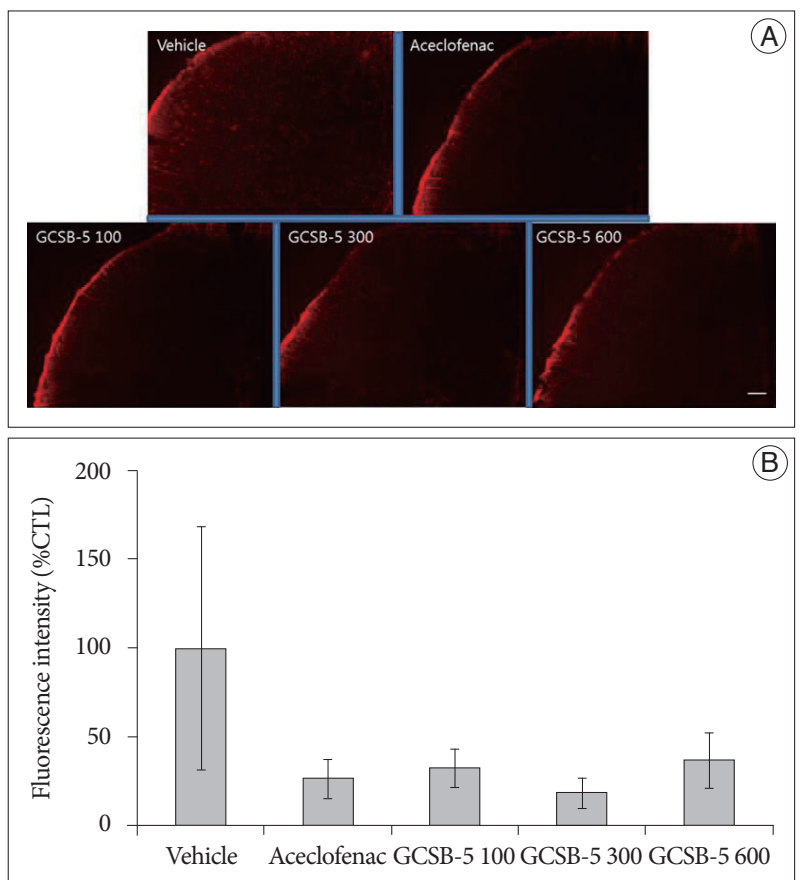

Fig. 4. Immunohistochemical expression of GFAP in ipsilateral L5 dorsal horns (A) and GFAP immunoreactivities versus vehicle controls (B) at 56 days after treatment commencement. A : GFAP-positive astrocyte numbers were lower in ipsilateral dorsal horns of the GCSB-5 300 group than in the aceclofenac or vehicle controls groups. B : Percentage decreases were greater in the GCSB- 5300 group than in the GCSB-5 100 , GCSB-5 600, aceclofenac or vehicle control groups. Results are presented as mean \pm SEM. Bar $=100 \mu \mathrm{m}$. GFAP : glial fibrillary acidic protein. 

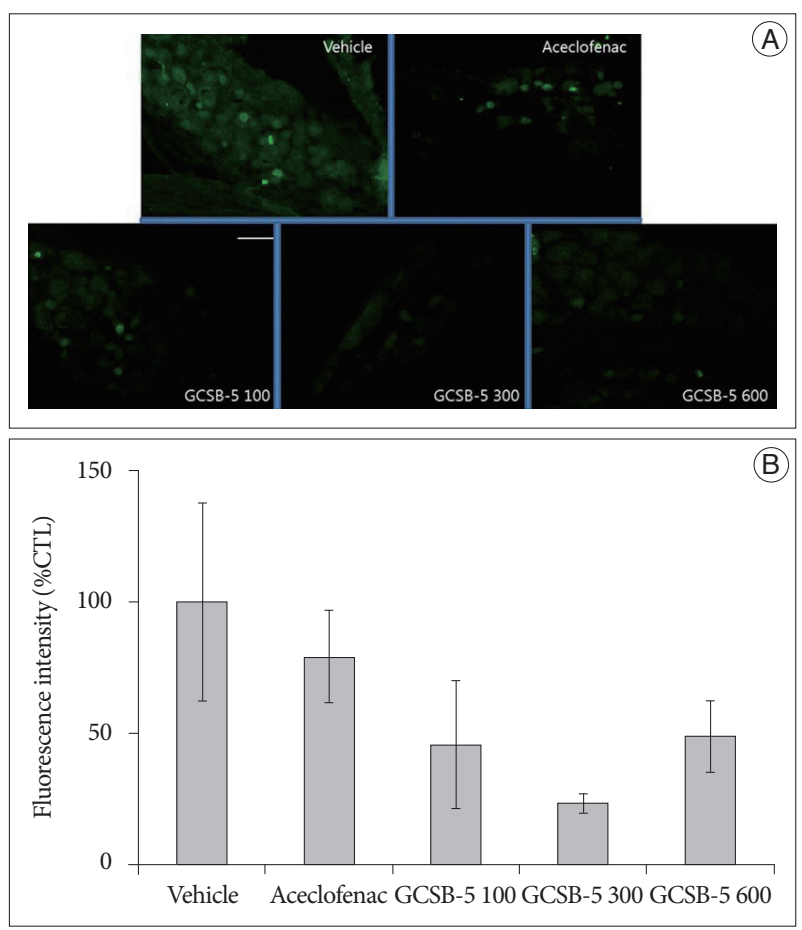

Fig. 5. Immunohistochemical expressions of CGRP in ipsilateral L5 dorsal root ganglions (DRGs) (A) and immunoreactivities of CGRP versus the vehicle controls (B) at 56 days after treatment commencement. A : CGRP expression was lower in ipsilateral DRGs in the GCSB-5 300 group than in the aceclofenac or vehicle control groups. $B$ : Percentage decreases were greater in the GCSB-5 300 group than in the GCSB-5 100, GCSB-5 600 , aceclofenac or vehicle control groups. Results are presented as mean \pm SEM. Bar $=100 \mu \mathrm{m}$. CGRP : calcitonin gene-related peptide.

significantly lower than in vehicle controls. Furthermore, the number of Iba1-positive microglia and GFAP-positive astrocytes in dorsal horns and CGRP and TRPV1 expressions in DRGs were lower in the three GCSB-5 groups than in the vehicle control group on TD56; however, these differences were not significant. IL- 6 and CX3CL1 mRNA expressions were unchanged by aceclofenac or GCSB- 5 versus controls.

Lumbar disc herniation is one of the most common causes of low back pain and sciatica, the latter of which is induced by mechanical and chemical factors ${ }^{2,21,32-34,48}$. It has been proposed that cytokines and chemokines play major roles in the chemical pathomechanisms of radicular pain ${ }^{1,8,17}$, and it is known that spinal cord and DRG responses play important roles. Furthermore, proinflammatory cytokine and neuroglial cell expressions in the spinal cord and DRG have been implicated in the generation of radicular pain ${ }^{31,41,45,47)}$. Radicular pain caused by lumbar disc herniation is characterized by hyperalgesia, spontaneous pain, and allodynia, and is normally treated using NSAIDs. However, the chronic nature of neuropathic pain requires the long-term use of NSAIDs, and this inseparable relationship unavoidably induces adverse drug reactions, such as, gastrointestinal and cardiovascular problems ${ }^{43}$.

GCSB- 5 is prepared from six herbs that are used to treat osteoarthritis in traditional East Asian medicine ${ }^{26}$. Thus, the safety of
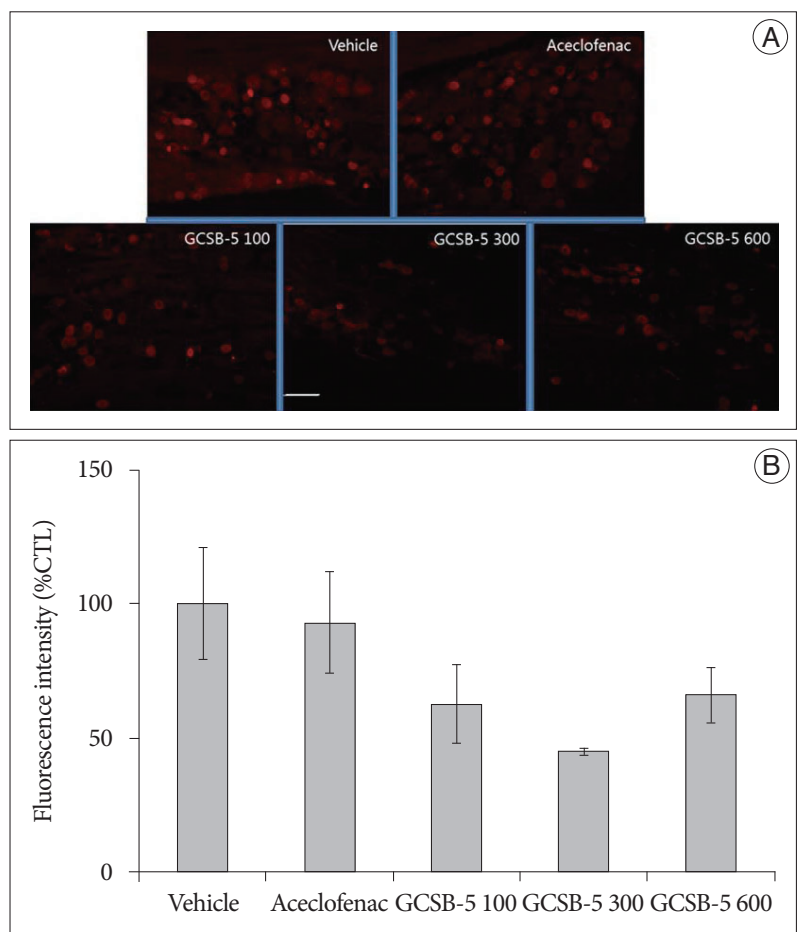

Fig. 6. Immunohistochemical expression of TRPV1 in ipsilateral L5 dorsal root ganglia (DRGs) (A) and TRPV1 immunoreactivities versus the vehicle controls (B) at 56 days after treatment commencement. A : TRPV1 expression was lower in the ipsilateral DRGs of the GCSB- 5300 group than in the aceclofenac or vehicle control groups. B : Percentage decreases in TRPV1 expression were greatest in the GCSB- 5300 group than in the GCSB-5 100, GCSB-5 600, aceclofenac or vehicle control groups. Results are presented as mean \pm SEM. Bar $=100 \mu \mathrm{m}$. TRPV1: transient receptor potential vanilloid 1.

these herbs is supported by long histories of human use. In the present study, we found GCSB-5 300 administration ameliorated mechanical allodynia induced by autologous nucleus pulposus implantation to a nerve root on TD56. In addition, the expressions of Iba1, GFAP, CGRP, and TRPV1 on TD56 showed a tendency to be reduced in the three GCSB-5 groups. Microglia are the resident macrophages of the CNS and contribute to the development of chronic neuropathic pain by releasing mediators, such as, proinflammatory cytokines and chemokines, that influence pain signaling ${ }^{28,42,46)}$. In previous studies, it has been suggested that nucleus pulposus application induces glial activity in spinal cords and that these activated glia play a crucial role in dorsal horn pain transmission ${ }^{16,29,35}$. In animal models of neuropathic pain, it has been reported that astrocytes are activated and express $\mathrm{GFAP}^{11,23,31)}$, and recently, it was suggested that injury-induced GFAP upregulation plays a role in the maintenance of neuropathic pain states ${ }^{23}$. In the present study, on TD56, numbers of Iba1-positive microglia and GFAP-positive astrocytes in dorsal horns showed a tendency to be reduced in the three GCSB- 5 groups, and similar results were also obtained for the expressions of CGRP and TRPV1. Recently, it was reported that the effects of nucleus pulposus on nerve roots are closely associated with cytokines such as TNF- $\alpha$, and cyclooxygenase- 2 

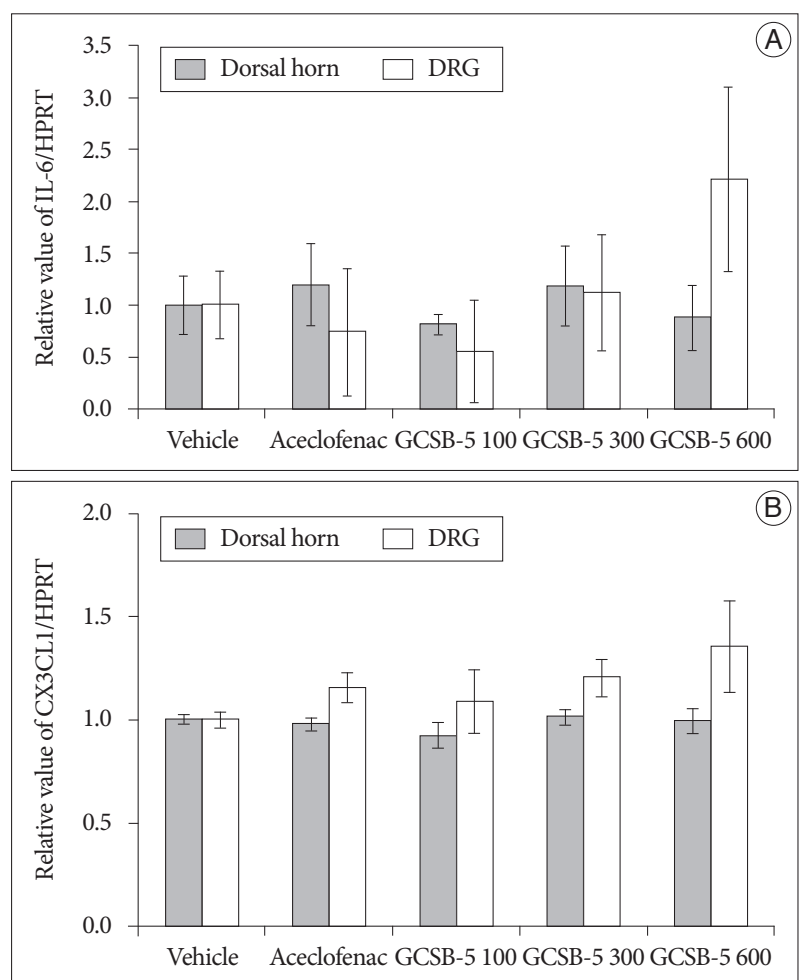

Fig. 7. Expressions of IL-6 (A) and CX3CL1 (B) mRNAs in ipsilateral L5 dorsal horns and dorsal root ganglia (DRGs). The mean expressions of the mRNAs of IL-6 and CX3CL1 in the aceclofenac and three GCSB-5 groups were not found to differ significantly from the vehicle control group at 56 days after treatment commencement. Results are presented as mean \pm SEM. IL-6 : interleukin-6, CX3CL1 : chemokine (C-X3-C motif) ligand 1/fractalkine, mRNA : messenger RNA.

$(\mathrm{COX}-2)^{30,33)}$. TNF- $\alpha$ induces the productions of inflammatory neuropeptides such as substance P (SP) and CGRP, and induces the release of SP and CGRP in the spinal dorsal horn from peripheral terminals ${ }^{9,13)}$. CGRP is a marker of sensory neurons involved mainly in pain perception. Moreover, TRPV1 receptors are molecular integrators of nociceptive stimuli at peripheral nerve endings, and spinal TRPV1 receptors have been shown to play important roles in nociceptive transmission modulation, especially under pathological conditions ${ }^{6,27,36,39)}$. These observed downregulations of microglia, astrocytes, CGRP, and TRPV1 with time after GCSB-5 treatment commencement follow a course similar to that of pain behavior attenuation, and thus, may be responsible for the analgesic effect of GCSB-5, which suggests GCSB-5 could provide a treatment for radicular pain caused by lumbar disc herniation by reducing neuroglial expression in spinal segments.

It was previously reported that GCSB-5 exhibited anti-inflammatory potential in a rat model of osteoarthritis. At a molecular level, GCSB-5 have been reported to inhibit nitric oxide production $^{10,44)}$, to reduce proinflammatory cytokine serum level ${ }^{49)}$, and to suppress COX-2 protein levels in macrophages ${ }^{22)}$, and in a recent animal study, GCSB-5 was found to have an anti-inflammatory effect on acute and chronic inflammation in arthritis ${ }^{7}$. In addition, in a study on its in vivo analgesic effects, it was concluded GCSB-5 ameliorated peripheral pain by increasing pain thresholds ${ }^{24)}$.

In the present study, we found no significant differences in the IL- 6 mRNA expression in dorsal horns and DRGs between the vehicle control group and the three GCSB-5 groups. Furthermore, CX3CL1 mRNA expressions in dorsal horns and DRGs in the vehicle control group and the three GCSB-5 groups were not significantly different. Cells of the nucleus pulposus have been shown to be capable of producing several cytokines and chemokines, including IL-1 $\alpha$, IL- $1 \beta$, TNF- $\alpha$, IL- 6 , IL- 8 , and IL$10^{1,18,19,37,40)}$. Chemokines constitute a family of proinflammatory cytokines with potent chemotactic activities for leukocytes in vitro and in vivo. They act at the initial stages of inflammatory response and draw inflammatory cells from blood into tissues. It has also been suggested chemokine production by herniated intervertebral disc tissue might explain the initiation of surrounding inflammation ${ }^{5)}$. Park et $\mathrm{al}^{35)}$, who used a nucleus pulposus implantation induced model of lumbar disc herniation, examined the longitudinal expression of CX3CL1 in spinal dorsal horns, and found CX3CL1 immunoreactivity gradually increased especially in laminae II and III of the ipsilateral dorsal horn. In addition, they observed strong CX3CL1 immunoreactivity in spinal neurons at 5 and 10 days after surgery but not observed in spinal cords at 10 and 30 days after surgery. In the present study, we examined IL- 6 and CX3CL1 mRNA expressions only at 56 days after treatment commencement, and did not monitor these expressions with respect to time. We believe this is why we observed no differences in IL-6 and CX3CL1 mRNA expressions between the three GCSB- 5 groups and the vehicle control group.

\section{CONCLUSION}

In conclusion, our results corroborate previous reports by showing that GCSB-5 administration ameliorates musculoskeletal pain-related behavior. To the best of our knowledge, this is the first report on neuroglial changes in lumbar spinal segments induced by oral GCSB- 5 administration in a rat model of radicular pain. Our results indicate GCSB-5 downregulates neuroglial activity in the spinal dorsal horn and the DRG expressions of CGRP and TRPV1, and suggest these downregulations attenuate radicular pain. More detailed studies are required to elucidate the mechanisms responsible for the observed molecular changes and the attenuation of radicular pain elicited by GCSB-5. In addition, we suggest clinical trials be conducted to investigate the efficacy and safety of Shinbaro ${ }^{\circledR}$ in patients with low back pain and/or radicular pain.

\section{- Acknowledgements}

This study was supported by Green Cross Corp. The funding source had no role in the design and conduct of the study; in the collection, analysis, and interpretation of the data; or in the preparation, review, or approval of the paper. 


\section{References}

1. Ahn SH, Cho YW, Ahn MW, Jang SH, Sohn YK, Kim HS : mRNA expression of cytokines and chemokines in herniated lumbar intervertebral discs. Spine (Phila Pa 1976) 27 : 911-917, 2002

2. Anzai H, Hamba M, Onda A, Konno S, Kikuchi S : Epidural application of nucleus pulposus enhances nociresponses of rat dorsal horn neurons. Spine (Phila Pa 1976) 27 : E50-E55, 2002

3. Aoki Y, Rydevik B, Kikuchi S, Olmarker K: Local application of disc-related cytokines on spinal nerve roots. Spine (Phila Pa 1976) 27 : 16141617,2002

4. Brisby H, Byröd G, Olmarker K, Miller VM, Aoki Y, Rydevik B : Nitric oxide as a mediator of nucleus pulposus-induced effects on spinal nerve roots. J Orthop Res 18 : 815-820, 2000

5. Burke JG, Watson RW, McCormack D, Dowling FE, Walsh MG, Fitzpatrick JM : Spontaneous production of monocyte chemoattractant protein-1 and interleukin- 8 by the human lumbar intervertebral disc. Spine (Phila Pa 1976) 27 : 1402-1407, 2002

6. Chen Y, Willcockson HH, Valtschanoff JG : Influence of the vanilloid receptor TRPV1 on the activation of spinal cord glia in mouse models of pain. Exp Neurol 220 : 383-390, 2009

7. Chung HJ, Lee HS, Shin JS, Lee SH, Park BM, Youn YS, et al. : Modulation of acute and chronic inflammatory processes by a traditional medicine preparation GCSB-5 both in vitro and in vivo animal models. J Ethnopharmacol 130 : 450-459, 2010

8. Cuellar JM, Montesano PX, Carstens E : Role of TNF-alpha in sensitization of nociceptive dorsal horn neurons induced by application of nucleus pulposus to L5 dorsal root ganglion in rats. Pain $110: 578-587,2004$

9. Ding M, Hart RP, Jonakait GM : Tumor necrosis factor-alpha induces substance $\mathrm{P}$ in sympathetic ganglia through sequential induction of interleukin-1 and leukemia inhibitory factor. J Neurobiol 28 : 445-454, 1995

10. Ge WH, Guo JY, Shen YJ, Chen ML, Shi SL, Han YH, et al. : [Effects of volatie oil of Schizonepeta tenuifolia Briq herb and Saposhnikovia divaricata Schischke root on proinflammatory cytokine expression and regulation]. Zhongguo Zhong Yao Za Zhi 32 : 1777-1179, 2007

11. Hatashita S, Sekiguchi M, Kobayashi H, Konno S, Kikuchi S : Contralateral neuropathic pain and neuropathology in dorsal root ganglion and spinal cord following hemilateral nerve injury in rats. Spine (Phila Pa 1976) $33: 1344-1351,2008$

12. Hong ND, Rho YS, Kim JW, Won DH, Kim NJ, Cho BS : Studies on the general pharmacological activities of Eucommia ulmoides Oliver. Korean J Pharmacogn $19: 102-110,1988$

13. Hua XY, Chen P, Fox A, Myers RR : Involvement of cytokines in lipopolysaccharide-induced facilitation of CGRP release from capsaicinsensitive nerves in the trachea : studies with interleukin-1beta and tumor necrosis factor-alpha. J Neurosci 16 : 4742-4748, 1996

14. Ida Y, Satoh Y, Katsumata M, Nagasao M, Hirai Y, Kajimoto T, et al. : Two novel oleanolic acid saponins having a sialyl Lewis X mimetic structure from Achyranthes fauriei root. Bioorg Med Chem Lett 8 : 2555 2558, 1998

15. Igarashi T, Kikuchi S, Shubayev V, Myers RR : 2000 Volvo Award winner in basic science studies : exogenous tumor necrosis factor-alpha mimics nucleus pulposus-induced neuropathology. Molecular, histologic, and behavioral comparisons in rats. Spine (Phila Pa 1976) 25 : 2975-2980, 2000

16. Ito T, Ohtori S, Inoue G, Koshi T, Doya H, Ozawa T, et al. : Glial phosphorylated p38 MAP kinase mediates pain in a rat model of lumbar disc herniation and induces motor dysfunction in a rat model of lumbar spinal canal stenosis. Spine (Phila Pa 1976) 32 : 159-167, 2007

17. Kallakuri S, Takebayashi T, Ozaktay AC, Chen C, Yang S, Wooley PH, et al. : The effects of epidural application of allografted nucleus pulposus in rats on cytokine expression, limb withdrawal and nerve root discharge. Eur Spine J 14 : 956-964, 2005
18. Kang JD, Georgescu HI, McIntyre-Larkin L, Stefanovic-Racic M, Donaldson WF 3rd, Evans CH : Herniated lumbar intervertebral discs spontaneously produce matrix metalloproteinases, nitric oxide, interleukin-6, and prostaglandin E2. Spine (Phila Pa 1976) 21 : 271-277, 1996

19. Kang JD, Stefanovic-Racic M, McIntyre LA, Georgescu HI, Evans CH : Toward a biochemical understanding of human intervertebral disc degeneration and herniation. Contributions of nitric oxide, interleukins, prostaglandin E2, and matrix metalloproteinases. Spine (Phila Pa 1976) 22 : 1065-1073, 1997

20. Kawakami M, Tamaki T, Hashizume H, Weinstein JN, Meller ST : The role of phospholipase A2 and nitric oxide in pain-related behavior produced by an allograft of intervertebral disc material to the sciatic nerve of the rat. Spine (Phila Pa 1976) 22 : 1074-1079, 1997

21. Kayama S, Konno S, Olmarker K, Yabuki S, Kikuchi S : Incision of the anulus fibrosus induces nerve root morphologic, vascular, and functional changes. An experimental study. Spine (Phila Pa 1976) 21 : 25392543, 1996

22. Kim BH, Park KS, Chang IM : Elucidation of anti-inflammatory potencies of Eucommia ulmoides bark and Plantago asiatica seeds. J Med Food $12: 764-769,2009$

23. Kim DS, Figueroa KW, Li KW, Boroujerdi A, Yolo T, Luo ZD : Profiling of dynamically changed gene expression in dorsal root ganglia post peripheral nerve injury and a critical role of injury-induced glial fibrillary acidic protein in maintenance of pain behaviors [corrected]. Pain 143 : 114-122, 2009

24. Kim HW, Kwon YB, Ham TW, Roh DH, Yoon SY, Han HJ, et al. : The antinociceptive and anti-inflammatory effect of ethylacetate extracts from Bang-Poong (Radix ledebouriellae) on the Freund's adjuvant-induced arthritis in rats. J Vet Sci 3 : 343-349, 2002

25. Kim JK, Park SW, Kang JW, Kim YJ, Lee SY, Shin J, et al. : Effect of GCSB5 , a herbal formulation, on monosodium iodoacetate-induced ssteoarthritis in rats. Evid Based Complement Alternat Med 2012 : 730907 , 2012

26. Kim SJ, Kim WR, Kim HS, Park HW, Cho YW, Jang SH, et al. : Abnormal spontaneous activities on needle electromyography and their relation with pain behavior and nerve fiber pathology in a rat model of lumbar disc herniation. Spine (Phila Pa 1976) 36 : E1562-E1567, 2011

27. Lappin SC, Randall AD, Gunthorpe MJ, Morisset V : TRPV1 antagonist, SB-366791, inhibits glutamatergic synaptic transmission in rat spinal dorsal horn following peripheral inflammation. Eur J Pharmacol 540 : 73-81, 2006

28. Marchand F, Perretti M, McMahon SB : Role of the immune system in chronic pain. Nat Rev Neurosci 6 : 521-532, 2005

29. Norimoto M, Sakuma Y, Suzuki M, Orita S, Yamauchi K, Inoue G, et al. : Up-regulation of pain behavior and glial activity in the spinal cord after compression and application of nucleus pulposus onto the sciatic nerve in rats. Asian Spine J 8 : 549-556, 2014

30. Ohtori S, Takahashi K, Aoki Y, Doya H, Ozawa T, Saito T, et al. : Spinal neural cyclooxygenase-2 mediates pain caused in a rat model of lumbar disk herniation. J Pain 5 : 385-391, 2004

31. Ohtori S, Takahashi K, Moriya H, Myers RR : TNF-alpha and TNF-alpha receptor type 1 upregulation in glia and neurons after peripheral nerve injury : studies in murine DRG and spinal cord. Spine (Phila Pa 1976) 29: 1082-1088, 2004

32. Olmarker K, Brisby H, Yabuki S, Nordborg C, Rydevik B : The effects of normal, frozen, and hyaluronidase-digested nucleus pulposus on nerve root structure and function. Spine (Phila Pa 1976) 22 : 471-475; discussion 476,1997

33. Olmarker K, Larsson $\mathrm{K}$ : Tumor necrosis factor alpha and nucleus-pulposus-induced nerve root injury. Spine (Phila Pa 1976) 23 : 2538-2544, 1998

34. Olmarker K, Rydevik B, Nordborg C : Autologous nucleus pulposus in- 
duces neurophysiologic and histologic changes in porcine cauda equina nerve roots. Spine (Phila Pa 1976) 18 : 1425-1432, 1993

35. Park HW, Ahn SH, Kim SJ, Seo JM, Cho YW, Jang SH, et al. : Changes in spinal cord expression of fractalkine and its receptor in a rat model of disc herniation by autologous nucleus pulposus. Spine (Phila Pa 1976) 36 : E753-E760, 2011

36. Patwardhan AM, Scotland PE, Akopian AN, Hargreaves KM : Activation of TRPV1 in the spinal cord by oxidized linoleic acid metabolites contributes to inflammatory hyperalgesia. Proc Natl Acad Sci U S A $106: 18820-18824,2009$

37. Rand N, Reichert F, Floman Y, Rotshenker S : Murine nucleus pulposusderived cells secrete interleukins-1-beta, -6 , and -10 and granulocytemacrophage colony-stimulating factor in cell culture. Spine (Phila Pa 1976) 22 : 2598-2601; discussion 2602, 1997

38. Sekiguchi M, Otoshi K, Kikuchi S, Konno S : Analgesic effects of prostaglandin E2 receptor subtype EP1 receptor antagonist : experimental study of application of nucleus pulposus. Spine (Phila Pa 1976) 36 : 1829-1834, 2011

39. Spicarova D, Palecek J : The role of the TRPV1 endogenous agonist NOleoyldopamine in modulation of nociceptive signaling at the spinal cord level. J Neurophysiol 102 : 234-243, 2009

40. Takahashi H, Suguro T, Okazima Y, Motegi M, Okada Y, Kakiuchi T : Inflammatory cytokines in the herniated disc of the lumbar spine. Spine (Phila Pa 1976) 21 : 218-224, 1996

41. Takeda M, Tanimoto T, Kadoi J, Nasu M, Takahashi M, Kitagawa J, et al. :
Enhanced excitability of nociceptive trigeminal ganglion neurons by satellite glial cytokine following peripheral inflammation. Pain 129 : 155166, 2007

42. Tsuda M, Inoue K, Salter MW : Neuropathic pain and spinal microglia : a big problem from molecules in "small" glia. Trends Neurosci 28 : 101107,2005

43. Vane J, Botting R : Inflammation and the mechanism of action of antiinflammatory drugs. FASEB J 1 : 89-96, 1987

44. Wang CC, Chen LG, Yang LL : Inducible nitric oxide synthase inhibitor of the Chinese herb I. Saposhnikovia divaricata (Turcz.) Schischk. Cancer Lett 145 : 151-157, 1999

45. Watkins LR, Maier SF : Beyond neurons : evidence that immune and glial cells contribute to pathological pain states. Physiol Rev 82 : 981-1011, 2002

46. Watkins LR, Maier SF : Glia : a novel drug discovery target for clinical pain. Nat Rev Drug Discov 2 : 973-985, 2003

47. Watkins LR, Martin D, Ulrich P, Tracey KJ, Maier SF : Evidence for the involvement of spinal cord glia in subcutaneous formalin induced hyperalgesia in the rat. Pain $71: 225-235,1997$

48. Yabuki S, Kikuchi S, Olmarker K, Myers RR : Acute effects of nucleus pulposus on blood flow and endoneurial fluid pressure in rat dorsal root ganglia. Spine (Phila Pa 1976) 23 : 2517-2523, 1998

49. Zhu J, Gao X, Xie WL, Jin YZ, Sun WJ : [Effect of geniposide on serum IL-1beta and TNF-alpha of rheumatoid arthritis rats]. Zhongguo Zhong Yao Za Zhi 30 : 708-711, 2005 